\title{
OP77
}

\section{CALIBRATION OF SPECTRORADIOMETERS USING TUNABLE LASERS}

\author{
Yuqin Zong et al.
}

DOI 10.25039/x46.2019.OP77

from

CIE x046:2019

\author{
Proceedings \\ of the \\ 29th CIE SESSION \\ Washington D.C., USA, June 14 - 22, 2019 \\ (DOI 10.25039/x46.2019)
}

The paper has been presented at the 29th CIE Session, Washington D.C., USA, June 14-22, 2019. It has not been peer-reviewed by CIE.

\section{(C) CIE 2019}

All rights reserved. Unless otherwise specified, no part of this publication may be reproduced or utilized in any form or by any means, electronic or mechanical, including photocopying and microfilm, without permission in writing from CIE Central Bureau at the address below. Any mention of organizations or products does not imply endorsement by the CIE.

This paper is made available open access for individual use. However, in all other cases all rights are reserved unless explicit permission is sought from and given by the CIE.

CIE Central Bureau

Babenbergerstrasse 9

A-1010 Vienna

Austria

Tel.: +43 17143187

e-mail: ciecb@cie.co.at

www.cie.co.at 


\title{
CALIBRATION OF SPECTRORADIOMETERS USING TUNABLE LASERS
}

\author{
Yuqin Zong, Ping-Shine Shaw, Joseph P. Rice, C. Cameron Miller \\ National Institute of Standards and Technology (NIST), Gaithersburg, Maryland, USA
}

yuqin.zong@nist.gov

DOI $10.25039 / \times 46.2019 .0 P 77$

\begin{abstract}
To shorten the long calibration chain when using the conventional source-based approach and therefore to reduce spectroradiometer's calibration uncertainty, we developed a detector-based approach to calibrate spectroradiometers directly against a transfer trap detector using a tunable laser. Two different detector-based methods are used for the calibration and their calibration results are in very good agreement with each other. The calibration result is also compared with that obtained using a working standard FEL lamp and the agreement is within the expanded uncertainties $(k=2)$ of the working standard FEL lamp. This detector-based approach enables new, independent realizations of spectral irradiance or radiance scales on spectroradiometers. Such spectroradiometers can be used as instrument-based primary or transfer standards to disseminate spectral irradiance or radiance scale with a smaller uncertainty.
\end{abstract}

Keywords: Detector-based Calibration, New Method, Spectroradiometer, Tunable Laser.

\section{Introduction}

Spectroradiometers are typically calibrated against broadband transfer-standard sources (e.g., deuterium lamps for the deep ultraviolet (UV) region and quartz tungsten halogen lamps for the UV, visible, and infrared (IR) regions). Using this conventional source-based calibration approach, however, the uncertainty in measured spectral irradiance or radiance responsivity of a spectroradiometer is dominated by the transfer-standard sources, which is typically about $1 \%$ in the visible and IR regions and much larger in the UV region. Such a high uncertainty arises because of a long chain of calibration in calibrating the transfer-standard sources. For example, when an FEL lamp is used for calibrating a spectroradiometer, the scale of the FEL lamp is derived and transferred in sequence from (1) primary cryogenic radiometer, (2) transfer trap detector, (3) reference field radiometer, (4) gold point blackbody, and (5) high temperature blackbody. To shorten the long calibration chain and therefore to reduce spectroradiometer's calibration uncertainty, we describe a new method for calibration of spectroradiometers directly against a transfer trap detector (i.e., bypassing most of the scale transfers mentioned above) using tunable lasers.

\section{The new calibration method}

The NIST automated calibration facility for calibration of detectors (Zong et al. 2012) (Woodward et al. 2018) is improved so it can also be used for calibration of spectroradiometers. A schematic for calibration of an irradiance spectroradiometer is shown in Figure 1. A fully automated tunable optical parametric oscillator (OPO) laser is used for this calibration, which has a tunable range from $210 \mathrm{~nm}$ to $2400 \mathrm{~nm}$. The repetition rate of the OPO is one kilohertz $(\mathrm{kHz})$ and its pulse width is five nanosecond (ns). The laser beam is guided into a $50 \mathrm{~mm}$ diameter integrating sphere using a multimode fiber so that the test spectroradiometer or standard trap detector is illuminated with a uniform beam. A laser spectrum analyser (LSA) having a spectral range of $180 \mathrm{~nm}$ to $1000 \mathrm{~nm}$ is used for measurement of the wavelength of OPO laser. The absolute wavelength standard uncertainty measured by the LSA is $5 \mathrm{pm}$ and relative wavelength standard uncertainty is $1 \mathrm{pm}$. The wavelength scale of the spectroradiometer is calibrated against the LSA with a standard uncertainty on the order of a picometer (Zong 2017). 
The calibration is based on the measurement of the total energy of a pulsed OPO train. This avoids measurement difficulties arising from the OPO's pulse-to-pulse fluctuations (more than $10 \%$ ) and extremely low duty cycle (approximately $10^{-5}$ ). The length of the pulsed OPO train is controlled by the laser shutter and varies from $1 \mathrm{~s}$ to $10 \mathrm{~s}$ depending on the laser power. A monitor detector is mounted near the test spectroradiometer or standard trap detector to measure the relative total energy of an OPO pulse train.

The standard trap detector and the irradiance probe of the test spectroradiometer are each mounted, in turn, to the center of the optical beam for the calibration using the substitution method. Two current integrators (also called charge amplifiers) (not shown) are used for simultaneous measurements of the total electric charge (unit: coulomb, symbol: C) from the standard trap detector and the monitor detector, respectively.

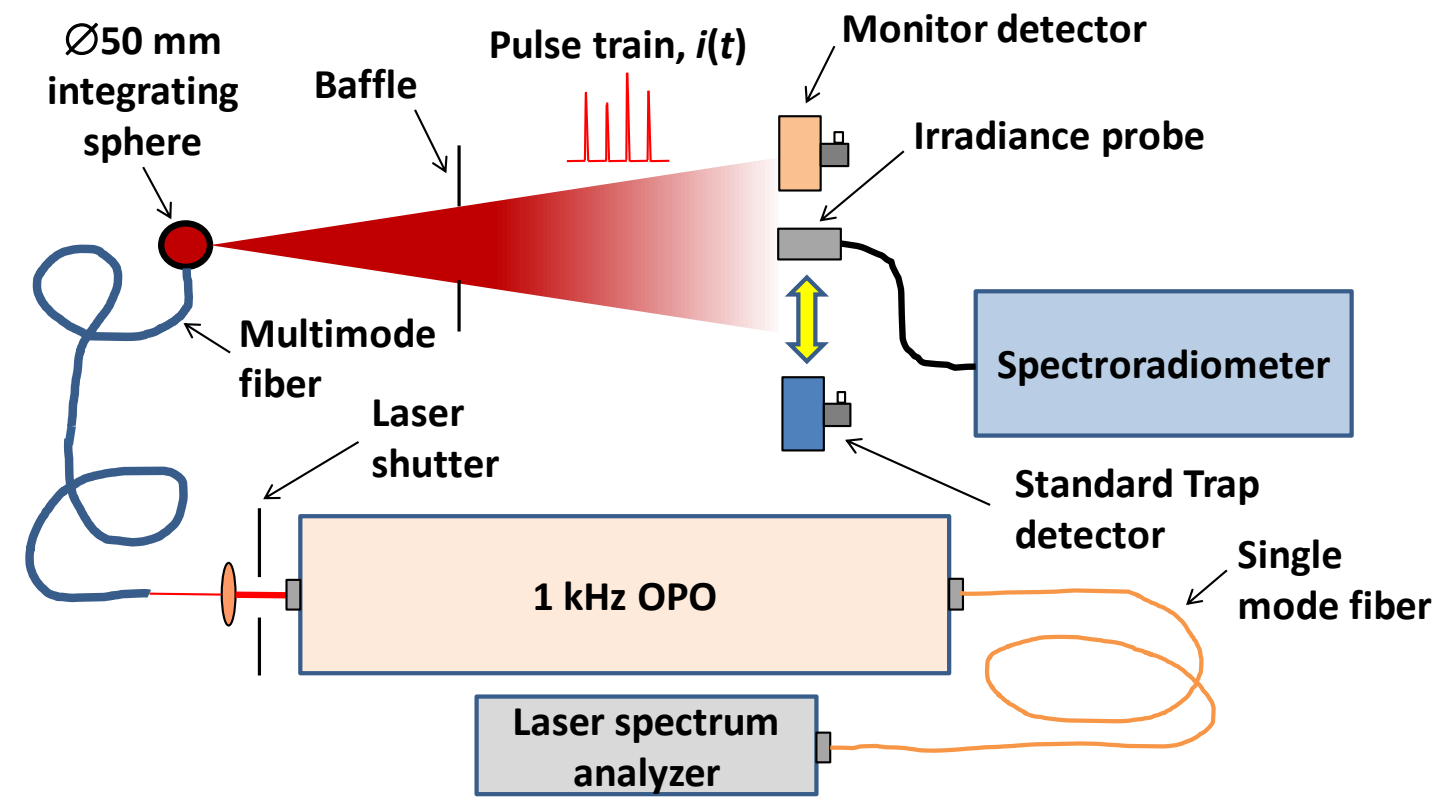

Figure 1 - Schematic for calibration of a spectroradiometer

Before the calibration, the stray light of the spectroradiometer is characterized and corrected (Zong et al. 2006) so that the spectroradiometer's response outside its bandpass is negligible. Two different methods, the slit scattering function (SSF) method and the line spread function (LSF) method, can be used for the calibration of spectroradiometers.

\subsection{SSF method (or overfill method)}

The SSF of an array spectroradiometer is the responsivity function of a pixel while the wavelength of the incident monochromatic source changes (Zong et al. 2006). If the SSF of a pixel is obtained by finely tuning the laser wavelength across the entire bandpass of the pixel, the sum of the response within the bandpass represents the signal when the spectroradiometer measures an imaginary broadband source with discrete wavelengths which spectrally overfills the bandpass of the pixel. Due to rapid rise-and-fall nature and narrow bandpass of an SSF, the wavelength of the laser should be tuned in a fine step (e.g., $0.1 \mathrm{~nm})$ and the bandwidth of the laser should be sufficiently narrow to minimize the convolution error arising from the finite bandpass and finite bandwidth of the laser. Using this method, each pixel is considered as a filter-radiometer.

The spectral irradiance responsivity at a pixel i for an equal energy source illuminant $\mathrm{E}, R_{\lambda, \mathrm{i}}$ (unit: count $\cdot \mathrm{s}^{-1} / \mathrm{W} \cdot \mathrm{m}^{-2} \cdot \mathrm{nm}^{-1}$ ), is given by

$$
R_{\lambda, \mathrm{i}}=\sum_{J \subset \mathrm{IB}}\left(\frac{y_{J}^{\mathrm{M}}}{Q_{J, \text { trap }}^{\mathrm{M}}} \cdot R_{\text {trap }}\left(\lambda_{J}\right) \cdot \Delta \lambda_{J, \text { step }}\right)
$$


where
$y_{J}^{\mathrm{M}}$
unit: count, is the signal of the pixel "i" at OPO wavelength $\lambda \mathrm{s}$, corrected by monitor signal and dark signal;
$Q_{J, \text { trap }}^{\mathrm{M}} \quad$ unit: C, is the electric charge of the standard trap detector at OPO wavelength $\lambda \mathrm{J}$, corrected by monitor signal and dark signal;
$R_{\text {trap }}\left(\lambda_{J}\right)$ unit: $\mathrm{A} / \mathrm{W} \cdot \mathrm{m}^{-2}$, is the responsivity of the standard trap detector at wavelength $\lambda_{\mathrm{J}}$;
$\Delta \lambda J$,step unit: $\mathrm{nm}$, is the wavelength interval of the scan.

As shown in Equation 1, the accuracy of wavelength interval, $\Delta \lambda_{J \text {,step }}$, is critical to achieve a small calibration uncertainty because $\Delta \lambda_{J \text {,step }}$ is small (e.g., $0.1 \mathrm{~nm}$ ).

\subsection{LSF method (or underfill method)}

LSF of an array spectroradiometer is the description of the response at every pixel to a particular incident monochromatic light (Zong et al. 2006). If the detector array has no or negligible pixel-to-pixel spatial non-uniformity of responsivity within the bandpass of the spectroradiometer, and has no or negligible dead region between adjacent pixels, which is generally true for many charge-coupled-device (CCD) instruments, a measured LSF, at pixel j with wavelength $\lambda_{\mathrm{j}}$, can be directly used for obtaining the spectral power responsivity of the spectroradiometer at wavelength $\lambda_{\mathrm{j}}, R_{\mathrm{j}}$ (unit: count $\cdot \mathrm{s}^{-1} / \mathrm{W} \cdot \mathrm{m}^{-2}$ ), by

$$
R_{\mathrm{j}}=\frac{\sum_{i \subset \mathrm{IB}} y_{i}^{\mathrm{M}}}{Q_{\mathrm{j}, \text { trap }}^{\mathrm{M}}} \cdot R_{\text {trap }}\left(\lambda_{\mathrm{j}}\right)
$$

where
$y_{i}^{\mathrm{M}}$ unit: count, is the signal of the pixel "i" within the bandpass, corrected by monitor signal and dark signal;
$Q_{\mathrm{j}, \text { trap }}^{\mathrm{M}} \quad$ unit: C, is the electric charge of the standard trap detector at OPO wavelength $\lambda_{\mathrm{j}}$, corrected by monitor signal and dark signal;
$R_{\text {trap }}\left(\lambda_{\mathrm{j}}\right) \quad$ unit: $\mathrm{A} / \mathrm{W} \cdot \mathrm{m}^{-2}$, is the responsivity of the standard trap detector at wavelength $\lambda_{\mathrm{j}}$.

Using this LSF method, the spectral width of a pixel of the spectroradiometer is underfilled by the bandwidth of the laser, and the output signals of the spectroradiometer on multiple pixels within the bandpass is deconvoluted to be at 1 pixel. Note in practice, it is not required to tune the laser wavelength to be exactly on a pixel. The spectral power responsivity at a pixel can be obtained by interpolation. The spectral irradiance responsivity of the spectroradiometer at pixel j with wavelength $\lambda_{\mathrm{j}}, R_{\lambda, \mathrm{j}}$ (unit: count $\cdot \mathrm{s}^{-1} / \mathrm{W} \cdot \mathrm{m}^{-2} \cdot \mathrm{nm}^{-1}$ ), can be converted from the obtained spectral power responsivity, $R_{\mathrm{j}}$, by

$$
R_{\lambda, \mathrm{j}}=R_{\mathrm{j}} \cdot \Delta \lambda_{\mathrm{j}} \cdot C_{\mathrm{j}}
$$

where
$\Delta \lambda_{\mathrm{j}}$
unit: $\mathrm{nm}$, is the spectral width of pixel $\mathrm{j}$ that can be determined by measuring pixel-to- pixel wavelength interval, $\Delta \lambda_{\mathrm{p}-\mathrm{p}, \mathrm{j}}$, at pixel $\mathrm{j}$;
$C_{\mathrm{j}}$
is the correction factor for non-uniformity of spectroradiometer's spectral power responsivity on pixel $\mathrm{j}$, which is the ratio of the average spectral power responsivity over the spectral width of pixel $\mathrm{j}$ to the spectral power responsivity at the center of pixel $\mathrm{j}$.

To convert the spectral power responsivity to spectral irradiance responsivity, the spectral width, $\Delta \lambda_{\mathrm{j}}$, commonly in the range of $1 \mathrm{~nm}$, must be determined with an uncertainty of picometers to achieve a small uncertainty for spectral irradiance responsivity (Zong 2017).

This LSF method does not require super fine scanning wavelength interval and super narrow bandwidth of the laser compared to the SSF method. Further, spectral irradiance responsivity at the particular wavelength can even be calibrated using a fixed wavelength laser (such as a He-Ne laser), which is very useful to check or monitor the change of the spectroradiometer in many applications. 


\section{Results of experimental calibration}

As an example, a CCD-array spectroradiometer with the spectral range from $300 \mathrm{~nm}$ to $1100 \mathrm{~nm}$ was calibrated using the new LSF methods described above. The calibration system was first evaluated for measurement repeatability. As shown in Figure 2, the measured spectroradiometer's responsivity at $558 \mathrm{~nm}$ varies within $0.1 \%$ with $5 \mathrm{~s}$ integration time for each data point.

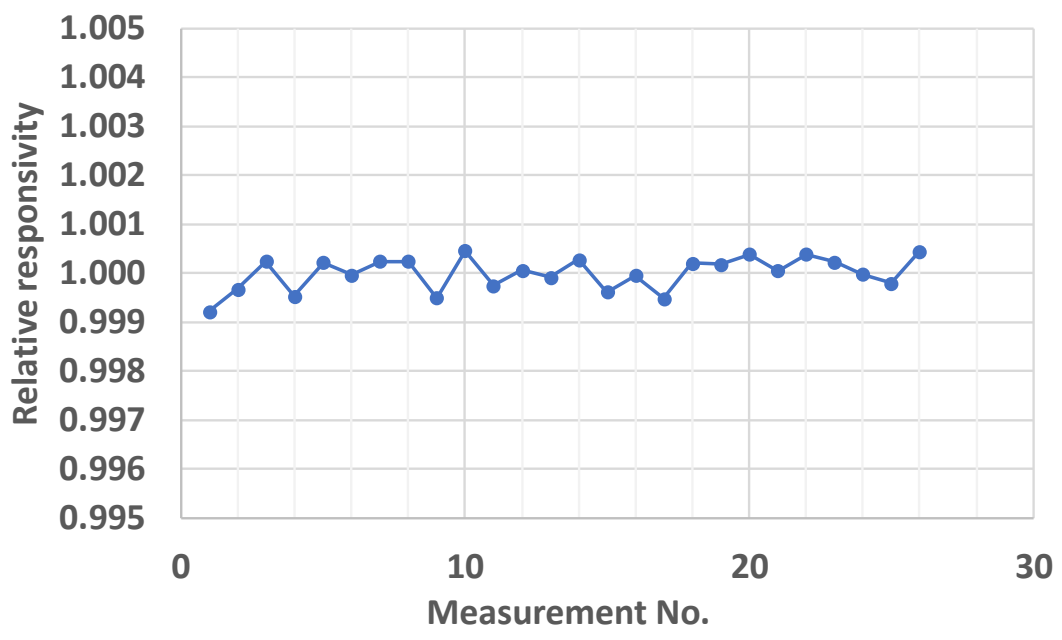

Figure 2 - Measurement repeatability of the spectroradiometer

The wavelength scale of the spectroradiometer is calibrated together with the spectral power responsivity with scanning interval of $1 \mathrm{~nm}$ from $350 \mathrm{~nm} 1000 \mathrm{~nm}$. This limited calibration spectral range is because a trap detector may be damaged by the deep UV light and it is not stable above $1000 \mathrm{~nm}$ without controlling its temperature. The determined pixel-to-pixel wavelength interval is shown in Figure 3. After smoothing, the error of the spectroradiometer's pixel-to-pixel wavelength interval is reduced to a few picometers.

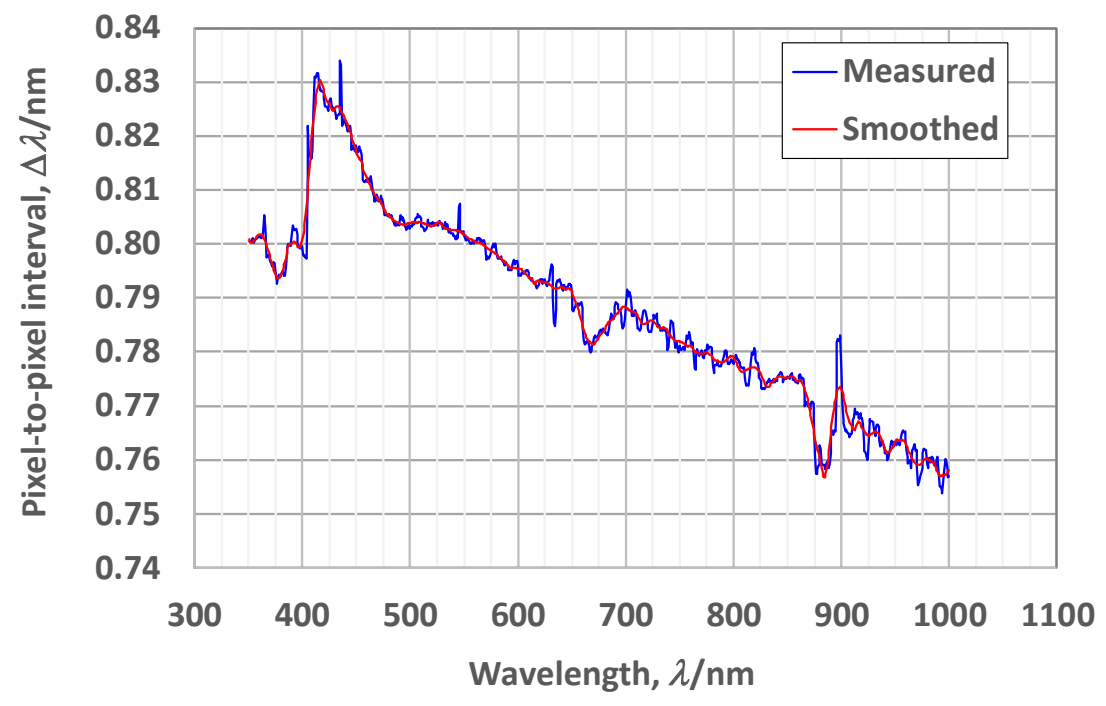

Figure 3 - Determined pixel-to-pixel wavelength interval

The spectroradiometer is also calibrated using the SSF method with a scanning interval of $0.1 \mathrm{~nm}$. The spectral irradiance responsivities obtained using the SSF method are compared with those obtained using the LSF method. As shown in the Figure 4, the difference in the calibration results is approximately $0.05 \%$. Thus, the two methods are equivalent in terms of the calibration results for this spectroradiometer. 


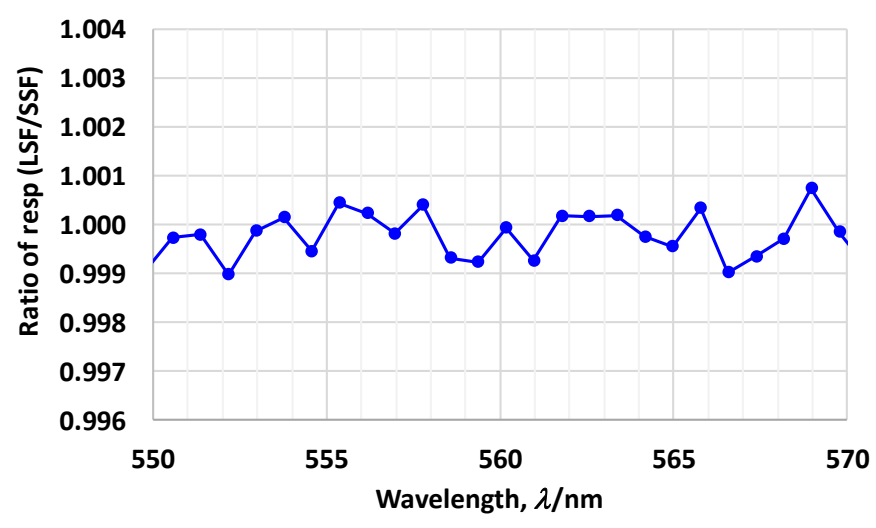

Figure 4 - Comparison of calibration results between using LSF method and using SSF method

The spectral irradiance results obtained using the detector-based LSF method are also compared to those obtained using the conventional source-based method. A spectral irradiance working standard FEL lamp is used for calibrating the spectroradiometer to obtain the spectral irradiance responsivities. Figure 5 shows the difference of the two calibration results. In the visible region from $450 \mathrm{~nm}$ to $700 \mathrm{~nm}$, the difference does not vary much with the wavelength and it is within the expanded uncertainty $(k=2)$ of the working standard FEL lamp. Outside $450 \mathrm{~nm}$ to $700 \mathrm{~nm}$, the fluctuation of the difference is caused by a number of factors such as a low signal-to-noise ratio with the LSF method due to spectroradiometer's low responsivity and low laser power, and a large pixel-to-pixel wavelength uncertainty, etc. The glitch around $410 \mathrm{~nm}$ is associated with the rapid change of the pixel-to-pixel wavelength interval. The deep valley around $940 \mathrm{~nm}$ is mainly due to very low signal-to-noise ratio $(<10: 1)$ with the LSF method resulting from the sharp absorption band of the spectroradiometer's optical fiber.

The output laser of the OPO is its idler above $710 \mathrm{~nm}$ and the laser bandwidth increases rapidly with the wavelength (Zong 2012). To reduce the wavelength uncertainty above $710 \mathrm{~nm}$, the OPO's signal wavelength and pump laser wavelength are measured using the LSA, and the OPO's idler wavelength is calculated using the measured signal wavelength and pump laser wavelength (as opposed to directly measurement).

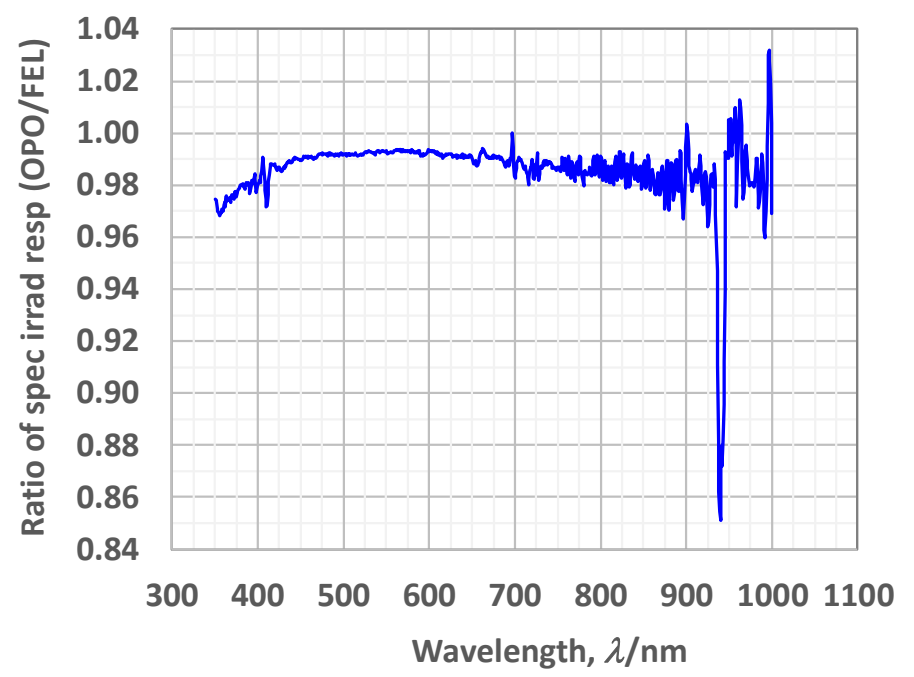

Figure 5 - Comparison of calibration results between using the detector-based LSF method and using the conventional source-based method. 


\section{Summary}

A detector-based approach for calibrating array spectroradiometers has been developed for reducing calibration uncertainties. Two different detector-based methods, SSF method and LSF method, are used for calibrating a CCD-array spectroradiometer and their calibration results are in very good agreement with each other. The calibration result using the LSF method is also compared with that obtained using a working standard FEL lamp and the agreement is within the expanded uncertainties $(k=2)$ of the working standard FEL lamp. The uncertainty of this detector-based calibration approach is being analysed and is expected to be much smaller than that of the conventional source-based calibration approach.

This newly developed approach enables a new, independent realization of spectral irradiance responsivity or radiance responsivity scales on spectroradiometers. Such spectroradiometers can be used as instrument-based primary or transfer standards to disseminate spectral irradiance or radiance scales with a smaller uncertainty. The detector-based calibration approach also eliminates the out-of-range stray-light error that is often the dominant source of calibration error in the UV region when a broadband standard source is used.

\section{Acknowledgements}

The authors want to thank their colleagues Steven Brown, Charles Gibson, John Woodward, Howard Yoon, Benjamin Tsai, and Maria Nadal for their support on this research.

\section{References}

NIST 2011. NIST Special Publication 250-89. Howard W. Yoon and Charles E. Gibson. NIST Measurement Services - Spectral Irradiance Calibrations. Gaithersburg: NIST. https://www.nist.gov/publications/sp250-spectral-irradiance-calibrations

Woodward, J. T. et al. 2018. Advances in tunable laser-based radiometric calibration applications at the National Institute of Standards and Technology, USA. Rev. Sci. Instrum., 89, 091301-1 - 091301-25.

ZONG, Y. et al. 2006. Simple Spectral Stray Light Correction Method for Array Spectroradiometers. Applied Optics, 45, 1111-1119.

ZONG, Y. et al. 2012. A New Method for Spectral Irradiance and Radiance Responsivity Calibrations using Kilohertz Pulsed Tunable Optical Parametric Oscillators. Metrologia, 49, S124-S129.

ZONG, Y. 2017. Wavelength Calibration Method for Spectroradiometers with Picometer Uncertainty. CIE, x044:2017, 736-739. 